\title{
Wavelet analysis on pressure stimulated currents emitted by marble samples
}

\author{
P. Kyriazis ${ }^{1,3}$, C. Anastasiadis ${ }^{1}$, D. Triantis ${ }^{1}$, and F. Vallianatos ${ }^{2}$ \\ ${ }^{1}$ Materials Technology Laboratory, Dept. of Electronics, Technological Educational Institution of Athens, Athens, Greece \\ ${ }^{2}$ Department of Natural Resources and Environment, Technological Educational Institution of Crete, Chania, Greece \\ ${ }^{3}$ School of Engineering and Design, Brunel University, Uxbridge, London, UK
}

Received: 17 July 2006 - Accepted: 3 October 2006 - Published: 10 October 2006

\begin{abstract}
This paper presents a wavelet based method of analysis of experimentally recorded weak electric signals from marble specimens which have undergone successive abrupt step loadings. Experimental results verify the existence of "memory effects" in rocks, as far as the current emission is concerned, akin to the "Kaiser effect" in acoustic emissions, which accompany rock fracturing. Macroscopic signal processing shows similarities and differences between the currents emitted during successive loading and wavelet analysis can reveal significant differences between the currents of each loading cycle that contain valuable information for the micro and macro cracks in the specimen as well as information for the remaining strength of the material. Wavelets make possible the time localization of the energy of the electric signal emitted by stressed specimens and can serve as method to differentiate between compressed and uncompressed samples, or to determine the deformation level of specimens.
\end{abstract}

\section{Introduction}

Wavelets analysis is an emerging area of applied mathematics that has innumerable applications in a variety of disciplines of science and engineering. In the last 20 years Wavelets are most commonly used as tools for signal processing, in a great variety of applications, from Medicine (mammograms and heart activity monitoring), to the compression of fingerprints for police databases and real-time industrial applications. However, Wavelet Transforms (WT), which are integral transforms using integration kernels called wavelets, originated in Geophysics in early 1980's for the analysis of seismic signals, in the outstanding work of Morlet (1982a, b). From that initial geophysical work, Earth Science has become the domain in which wavelets dominate the last decade with worth mentioning papers analyzing transient and spatially localized phenomena as seafloor bathymetry atmospheric turbulence, marine and seismic or earthquake precursory phenomena, the most representative of which are presented by Foufoula and Kumar (1994).

Electric signals emitted by geomaterials (rocks) have been systematically studied and outstanding works are available in bibliography. More precisely experiments have been conducted on rock specimens suggesting that the electric signals are produced by the piezoelectric effect due to the presence of quartz (Nitsan, 1977), electrokinetic effect due to water movement (Ishido and Mizutani, 1981), point defects (Hadjicontis and Mavromatou 1994), emission of electrons (Brady and Rowell, 1986), moving charged dislocations (MCD) (Vallianatos and Tzanis, 1998; Vallianatos et al., 2004). In recent laboratory experiments and observations electric signals were recorded after having applied various stress modes (uniaxially) on marble rocks (Stavrakas et al., 2003; Anastasiadis et al., 2004; Stavrakas et al., 2004; Triantis et al., 2006).

The technique that allows the recording of low currents emitted after having applied uniaxial compressional stress on samples is referenced as Pressure Stimulated Current technique (Anastasiadis et al., 2004), named after the emitted currents, that are referred as Pressure Stimulated Currents (PSC).

In this work weak electric current signals emitted by marble specimens subjected to uniaxial stress up to fracture and failure are thoroughly examined. The recorded signals are then analyzed using Continuous Wavelet Transform (CWT).

Correspondence to: $\mathrm{P}$. Kyriazis

(Panagiotis.Kyriazis@brunel.ac.uk)

Published by Copernicus GmbH on behalf of the European Geosciences Union. 


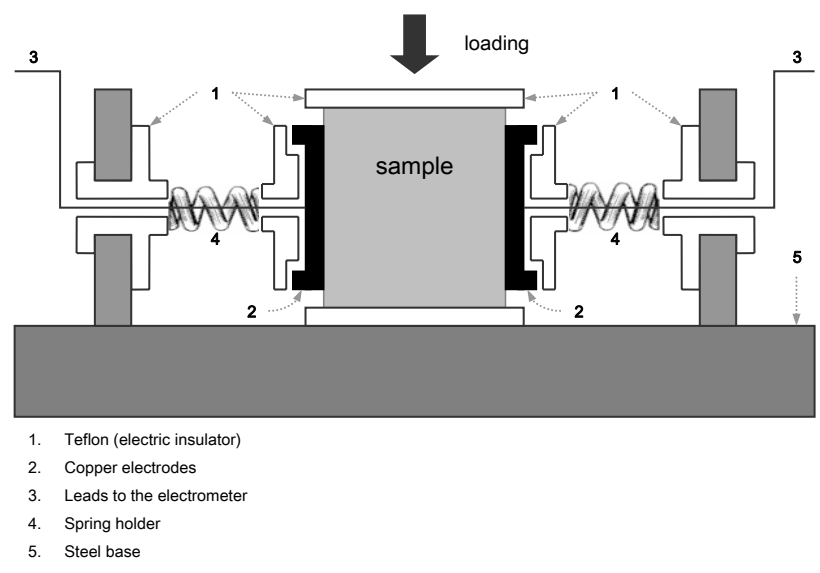

Fig. 1. Diagram of the experimental setup - electrodes and teflon insulators placement for weak current measurement from marble sample under uniaxial compressional stress.

\section{Experimental procedure}

Marble is a geomaterial whose physical and chemical properties have been thoroughly presented in the previous works. The marble samples under examination were collected from Mt. Penteli (Dionysos) and are mainly composed of calcite (98\%) and other minerals like muscovite and chlorite. The specimens are cubical (dimensions: $50 \mathrm{~mm} \times 50 \mathrm{~mm} \times 50 \mathrm{~mm}$ ) and their fracture limit is 58 $65 \mathrm{MPa}$. Marble contains approximately $0.2 \%$ of quartz, its density is $2.7 \mathrm{gr} / \mathrm{cm}^{3}$ and its porosity is low $(0.4 \%)$ (Kleftakis et al., 2000).

The experiment is conducted inside a Faraday shield in order to minimize the electromagnetic interference and immunize the recorded electric signals against the ambient electromagnetic noise. A hydraulic press (EnerpacRC106) is used as loading machine to uniaxially stress the marble samples that are placed on a stainless steel base. The marble samples are sited between two thin Teflon plates in the direction of the applied stress to electrically insulate them. The applied stress is measured by a manometer, while a pair of copper electrodes is attached to the marble sample using conductive paste, which serves as interface between the two materials (marble - copper). The placement of electrodes (perpendicular to the direction of the applied stress) as well as the whole experimental setup is depicted in Fig. 1. The measuring system is consisted of a sensitive electrometer (Keithley 617), which is capable of measuring low currents, like those recorded in the experiment, and stores them in a computer though GPIB (IEEE 488) interface.

The experiment was held in three stages. The marble samples were subjected to three loading cycles of the same stress level. After each stress cycle there was a rest period for the sample to relax. This rest period was not too long and the sample remained in the same position between successive
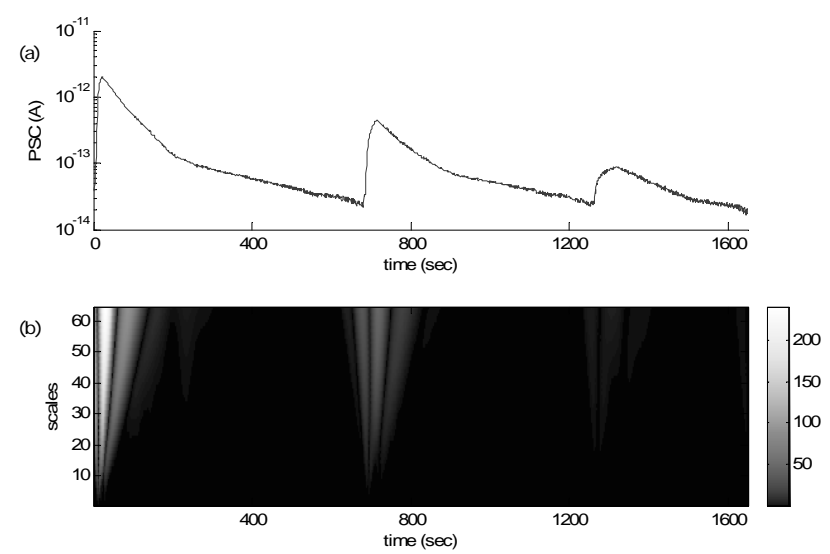

Fig. 2. (a) Pressure Stimulated Current recordings from the three successive loading cycles merged in the same time axis, (b) Timescale analysis (scalogram) of the electric signal, resulting from CWT.

loadings, in order to avoid the elimination of the memory effects (Lavrov, 2005).

Abrupt step-wise stress was chosen as the appropriate loading scheme for the three loading cycles. The stress rate $(d \sigma / d t)$ was quite high, of about $4 \mathrm{MPa} / \mathrm{sec}$ and the maximum stress applied to the sample was about 40MPa. Baring in mind that the failure limit for the samples is $58-65 \mathrm{MPa}$, the applied stress exceeded $60 \%$ of the failure stress. The reason for leading the sample near to failure limits was the creation of microcracks and therefore the recording of emitted PSC that are shown in Fig. 2a. Note that PSC are presented successively in the time axis despite the fact that after the completion of the relaxation processes of each cycle, the material was left to rest for almost an hour.

\section{Results and discussion}

\subsection{Macroscopic analysis and "memory effect"}

The Pressure Stimulated Currents emitted by the sample in each loading cycle show clearly that the relationship between the applied stress and the emitted current is not linear, but on the contrary a transient phenomenon is observed. Speaking in terms of signal processing the system, which is the marble sample in our case, responds not only according to the input, i.e. the applied stress, but also according to its previous state, i.e. the number of previous equi-loading cycles. This ability of marble and generally rocks to retain "imprints" from previous treatments and to reproduce information about these treatments under certain conditions is called "memory" in analogy to the memory of human beings. Particular expressions are referenced in literature as "memory effects". The earliest manifestation of such phenomena is 
Table 1. The parameters that arise from fitting of the PSC signals in every loading cycle according to equation (1) and the correlation coefficient showing the accuracy of the fitting.

\begin{tabular}{cccccc}
\hline PSC signals & $\mathrm{A}_{1}\left[\times 10^{-13}\right]$ & $\tau_{1}$ & $\mathrm{~A}_{2}\left[\times 10^{-14}\right]$ & $\tau_{2}$ & Correlation coef \\
\hline 1st cycle & 26.34 & 65.57 & 21.11 & 313.77 & 0.99 \\
2nd cycle & 6.08 & 93.81 & 12.88 & 355.75 & 0.98 \\
3rd cycle & 1.33 & 160.41 & 5.05 & 438.02 & 0.92 \\
\hline
\end{tabular}

the acoustic emission memory effect known also as "Kaiser effect" named after J. Kaiser who first observed it.

In this work an experimental verification of the existence of "memory effects" in the PSC emission, by analogy to the memory effects in acoustic emissions of a brittle rock (marble) during fracture is presented for the first time.

More specifically the peaks of the emitted PSCs are significantly lower in each loading cycle. Furthermore the response of the sample to loading is slower, as the time interval from the moment that the stress is applied to the moment that the PSC peak is observed is longer in every loading cycle. The aforementioned quantitative results show that the reaction of a pristine marble sample to uniaxial stress is initially intense, while the same sample reacts mildly in the following loading cycles, provided that the stress characteristics are the same.

Another key point for the emitted PSC is their relaxation. The relaxation interval is longer in the first loading and decreases in each loading cycle. The relaxation process that seems to govern the PSC emissions can be separated into two stages (fast process and slow process accordingly). In this experiment the same mechanisms seem to rule the relaxation process in all loading cycles. These processes can be mathematically expressed with the following equation,

$I(t)=\left\{\begin{array}{c}A_{1} \cdot \exp \left(-\frac{t}{\tau_{1}}\right) \text { for } t>t_{m} \\ A_{2} \cdot \exp \left(-\frac{t}{\tau_{2}}\right) \text { for } t>>t_{m}\end{array}\right.$

where $t_{m}$ is the moment that the PSC becomes maximum, $\tau_{1}$ and $\tau_{2}$ the relaxation time constants for the two processes and $A_{1}, A_{2}$ constant numbers. By fitting the PSC according to the above mentioned equation we get the coefficients as presented in Table 1.

The resulting fitted graphs are given in Fig. 3 in the same time axis, getting as starting point the moment that the stress was applied in each cycle, in order to be able to evaluate and compare the emitted PSC.

\subsection{Wavelets as a tool of analysis}

Wavelets is a modern tool for signal processing that is able to represent a process by an infinite series expansion of dilated and translated versions of a mother wavelet, each multiplied

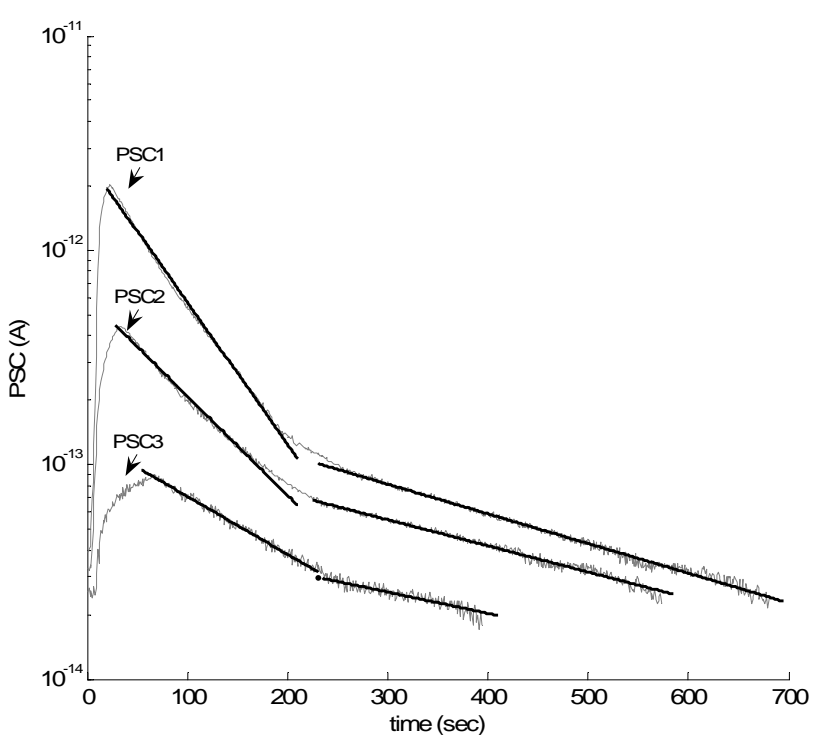

Fig. 3. Pressure Stimulated Currents emitted during each loading cycle, fitted according to Eq. (1). Starting time is the moment of the application of stress in each cycle.

by an appropriate coefficient. The coloration of the time scale plane according to the value of the aforementioned coefficients is called a scalogram, it was introduced by Flandrin (1988) and it is the result of the wavelet analysis. Wavelet Transform is superior to Fourier Transform (FT), because of its advanced features, which are concisely discussed in the following paragraphs in order to justify and explain why it was chosen as tool of analysis for the PSC.

The main advantage of analysing a signal with wavelets as the analysing kernels is that it allows the studying of features of the signal locally with a detail matched to their scales, i.e. broad features of the signal on a large scales and fine features on small scales.

This advantage of WT is inherent as each wavelet function, which is used for analysis, is localized in both time and frequency space (Farge, 1992). Therefore it is more suitable for transient, non-stationary and time-varying phenomena, and for signals that have short lived transient components at different scales, such as those examined in this paper. On the contrary FT, which uses sinusoidal waves, is better for peri- 
(a)

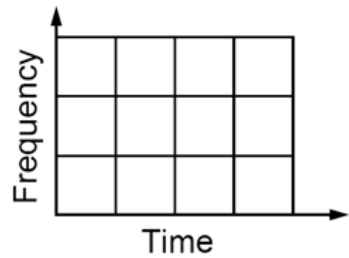

(b)

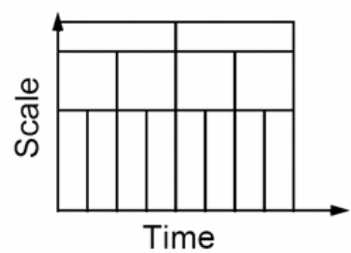

Fig. 4. Different windowing approach between (a) Short Time Fourier Transform (or Windowed Fourier Transform) and (b) Wavelet Transform.

odic and stationary signals. The localization of the energy of the PSC (white region of the scalogram) that is depicted in Fig. $2 b$ proves the suitability of wavelets for this analysis. Even the amelioration of FT is less accurate tool for the localization of the energy of a signal, as it is thoroughly discussed by Kaiser (1994).

Additionally, Wavelet Transform represents better the details of the signal (high frequency) and the approximation or trend (low frequency) simultaneously, because WT involves windowing technique with variable-sized regions (Fig. 4b), while windowing in Short Time Fourier Transform (STFT) is performed with window of particular size (Fig. 4a). The fact that the phase space is layered with resolution cells of varying dimensions does not break the uncertainty principle, which states that arbitrary high precision in both time and frequency cannot be achieved, because the dimensions of the cells are functions of scale such that they have a constant area. The above described idea of varying windowing technique is presented in Fig. 4b. Finally, the phenomenon of current emission has finite energy, and thus wavelet series expansion offers optimal approximation of the original signal, in least square sense.

\subsection{Selection of the mother wavelet for the analysis of PSC}

The selection of the mother wavelet is of paramount importance, as not only each of the wavelet families, but even among the members of the same family there are differences that may contribute substantially to the results of WT. For the analysis of the PSCs the third Daubechies wavelet (Daubechies, 1992) was chosen after considering the following factors.

1. The Daubechies wavelets are orthogonal and can be used as mother wavelets for both Continuous Wavelet Transform (CWT) and Discrete Wavelet Transform (DWT) analysis. In this work the results presented yield from CWT, which is possible for both orthogonal and non-orthogonal wavelets, however future work may involve DWT, which is faster and better for real-time applications.

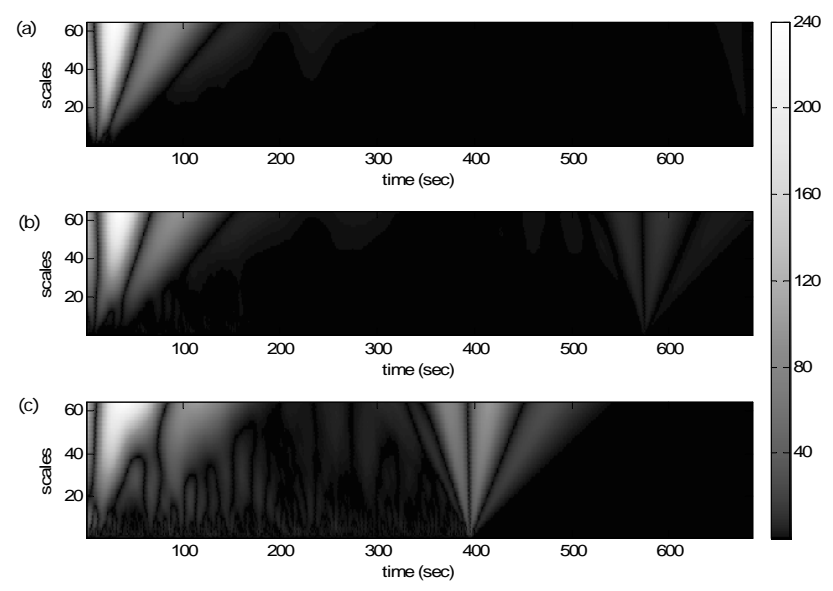

Fig. 5. Scalograms yielding from the CWT of the PSC emitted by the sample in the (a) 1st loading cycle, (b) 2nd loading cycle and (c) 3rd loading cycle (small coefficients dark regions, large coefficients bright regions).

2. Daubechies wavelets are complex functions, i.e. include both amplitude and phase information

3. Daubechies wavelet used in this analysis is the third of the family, which means that is narrow enough to provide good time resolution (Torrence, 1998). Note that the first wavelet of the Daubechies family is the Haar wavelet and this is the narrowest of all.

4. The shape of Daubechies wavelet is not symmetrical and not smooth, on the contrary it is quite sharp and thus is suitable for analysis of signals with irregularities as those examined here. A key feature as far as the shape of Daubechies wavelets is concerned is that they have fractal structure (self-similarity) as discussed in Daubechies 1992 and that was proved an important factor that is discussed in the following section.

\subsection{Results of CWT to PSC and discussion}

The results of the application of CWT to the PSC, using the third Daubechies as mother wavelet, are presented in Fig. 5. Note that PSC yielding from each loading cycle is separately analyzed.

The analysis of the PSC of the first loading cycle (see Fig. 5a) shows significantly large coefficient values at large scales (i.e. low frequency) while high frequency coefficients are negligible.

The scalogram that yields from the CWT of the second loading cycle PSC (see Fig. 5b), shows again appreciable large scale components, but these are spread slightly different in time compared to those of the first PSC scalogram. In the second loading cycle small scale components have such values that although they exist, they are discernible in the scalogram. 
The CWT analysis of the third PSC signal is the most impressive, as it gives a completely different scalogram (see Fig. 5c). In this scalogram small scale components of the signal are prevalent. Low frequency components still exist but they seem to be surpassed by dominant high frequency components.

The analysis of the latter signal revealed a fractal like scalogram (self-similarity between scales), something that is not probable to be coincidence, as fracture of a rock has been proved to be accompanied by fractal acoustic emissions (Mogi, 1962), while the acoustic emissions prior to the failure of a rock also follows power law (fractal) spatial distribution (Hirata et al., 1987; Turcotte et al., 2003).

In this paper the fractal form of the scalogram that corresponds to PSC yielding from heavily stressed marble samples to the failure limits are presented for the first time. This is very important because it shows not only the level of deformation of a geomaterial under stress but also the remaining strength of the material, which is very useful for applications in constructions and civil engineering.

\section{Conclusions}

The existence of "memory effects" in the emission of weak electric currents (PSC) by marble samples that were subjected to uniaxial compressional stress was experimentally verified. The wavelet based analysis was used alongside with curve fitting to macroscopically analyze the recorded signals from each loading cycle and useful remarks were made concerning the localization of the energy of the signal as well as the macroscopic resemblance of the signals of each loading cycle. Finally the wavelet analysis and more specifically the CWT, with Daubechies third wavelet as mother wavelet, was used to analyze the details of the signals recorded in each loading cycle and the resulting scalograms clearly depicted the differences which resulted from the successive loading of the samples near to fracture limit.

The above mentioned contribute to a better understanding of the electric effects that accompany deformation processes of a material and wavelet analysis seems to be a promising tool for the processing of Pressure Stimulated Currents and the characterization of a material according to its remaining strength.

Acknowledgements. This work is supported by the project ARCHIMEDES II: "Support of Research Teams of Technological Educational Institute of Athens", sub-project entitled "The electric behavior of geo-materials" in the framework of the Operational Program for Education and Initial Vocational Training. The project is co-funded by the European Social Fund and National Resources.

\section{References}

Anastasiadis, C., Triantis, D., Stavrakas, I., and Vallianatos, F.: Pressure Stimulated Currents (PSC) in marble samples after the application of various stress modes before fracture, Annals of Geophysics, 47, 21-28, 2004.

Brady, B. T. and Rowell, G. A.: Laboratory investigation of electrodynamics of rock fracture, Nature, 321, 448-492, 1986.

Daubechies, I.: Ten Lectures on Wavelets, Society for Industrial and Applied Mathematics (SIAM), 1992.

Farge, M.: Wavelet Transforms and their applications to turbulence, Annu. Rev. Fluid Mech., 24, 395-457, 1992.

Flandrin, P.: Time-frequency and time-scale, IEEE Fourth annual ASSP Workshop on Spectrum estimation and modelling, Minneapolis, Minnesota, 77-80, 1988.

Foufoula-Georgiou, E. and Kumar, P.: Wavelets in Geophysics, Academic Press, 1994.

Hadjicontis, V. and Mavromatou, C.: Transient electric signals prior to rock failure under uniaxial compression, Geophys. Res. Lett., 21, 1687-1990, 1994.

Hirata, T., Satoh, T., and Ito, K.: Fractal structure of spatial distribution of microfracturing in rock, Geophys. J. R. Astr. Soc., 90, 369-374, 1987.

Ishido, T. and Mizutani, H.: Experimental and Theoritical basis of electrokinetic phenomena in rock-water systems and its applications to geophysics, J. Geophys. Res., 86, 1763-1775, 1981.

Kaiser, G.: A Friendly Guide to Wavelets, Boston, Birkhäuser, 1994.

Kleftakis, S., Agioutantis, Z., and Stiakakis, C.: Numerical Simulation of the uniaxial compression test including the specimenplaten interaction, Computational methods for shell and spatial structures, IASS-IACM, 2000.

Lavrov, A.: Fracture - induced Physical Phenomena and Memory Effects in Rocks: A Review, Strain 41(4), 135-149, 2005.

Mogi, K.: Study of elastic shocks caused by the fracture of heterogeneous materials and its relations to earthquake phenomena, Bull. Earthq. Res. Inst., 40, 125-173, 1962.

Morlet, J., Arens, G, Fourgeau, E., and Giard, D.: Wave propagation and sampling theory - part 1: complex signal and scattering in multilayered media, Geophysics , 47(2), 203-221, 1982.

Morlet, J., Arens, G, Fourgeau, E., and Giard, D.: Wave propagation and sampling theory - part 2: sampling theory and complex waves, Geophysics, 47(2), 222-236, 1982.

Nitsan, U.: Electromagnetic emission accompanying fracture of quartz-hearing rocks, Geophys. Res. Lett., 4, 333-337, 1977.

Stavrakas, I., Anastasiadis, C., Triantis, D., and Valliantos, F.: Piezo-Stimulated currents in marble samples: Precursory and concurrent - with - failure signals, Nat. Hazards Earth Syst. Sci., 3, 243-247, 2003, http://www.nat-hazards-earth-syst-sci.net/3/243/2003/.

Stavrakas, I., Triantis, D., Agioutantis, Z., Maurigiannakis, S., Saltas, V., Vallianatos, F., and Clarke, M.: Pressure stimulated currents in rocks and their correlation with mechanical properties, Nat. Hazards Earth Syst. Sci., 4, 563-567, 2004, http://www.nat-hazards-earth-syst-sci.net/4/563/2004/.

Torrence, C. and Compo, G.: A practical guide to wavelet analysis, Bull. Amer. Meteorol. Soc., 79, 61-78, 1998.

Edited by: P. F. Biagi

Reviewed by: G. Nover 
Triantis, D., Stavrakas, I., Anastasiadis, C., Kyriazopoulos, A., and Vallianatos, F.: An analysis of Pressure Stimulated Currents (PSC), in marble samples under mechanical stress, Physics and Chemistry of the Earth, parts A/B/C, 31, 4-9, Recent Progress in Seismo Electromagnetics and Related Phenomena, 234-239, 2006.

Turcotte, D. L., Newman, W. I., and Shcherbakov, R.: Micro and macroscopic models of rock fracture, Geophysis. J. Int., 152, 718-728, 2003.
Vallianatos, F. and Tzanis, A.: Electric current generation associated with the deformation rate of a solid: Preseismic and coseismic signals, Phys. Chem. Earth, 23, 933-938, 1998.

Vallianatos, F., Triantis, D., Tzanis, A., Anastasiadis, C., and Stavrakas, I.: Electric Earthquake Precursors: From Laboratory Results to Field Observations, Phys. Chem. Earth, 29, 339-351, 2004.

Yamshchikov, V. S., Shkuratnik, V. L., and Lavrov, A. V.: Memory Effects in rocks (review), J. Mining Sci., 30, 5, 463-473, 1994. 